\title{
Recipes for success
}

\author{
Scientific and technological advances can only be achieved through careful experimentation, but what \\ has been discovered often overshadows how the discovery was made. However, there are a variety of \\ mechanisms, old and new, for the sharing of practical expertise.
}

No one can seriously doubt that scientific discovery is tightly bound up with the processes for making those discoveries. However far-sighted a scientist may be they must wait for technical advances to open up the vistas into which they can stare. Conversely, the arrival of a new technique will open up undreamed of avenues for its use.

A case in point is the CRISPR-Cas approach to genome editing. Clustered regularly interspaced short palindromic repeats (CRISPRs) were identified in prokaryotic genome sequences in the early 2000s and the involvement of CRISPRassociated (Cas) proteins in defence against prokaryotes emerged a few years later. Around 2012-2013 the potential of this system for modifying the genomes of animals and plants in a controlled way was demonstrated and since then there has been an explosion in its use both for 'pure' research and practical applications.

The potential of CRISPR-Cas for biotechnology has sparked public debate over the ethics of its use both in the creation of new crop varieties and the editing of animal (including human) genomes at the embryo stage. Federally funded research on human embryos is currently banned in the USA but an application has recently been made in the UK to the Human Fertilisation and Embryology Authority (HFEA) to use CRISPR-Cas in human embryos (http:// go.nature.com/kfsZ7I).

CRISPR-Cas is no less useful for plant science. In this issue of Nature Plants we publish two Brief Communications (15144 and 15145) deploying it against viral infections to create Nicotiana benthamiana plants resistant to specific geminiviruses. Dandan Zhang, Zhenxiang Li and Jian-Feng Li discuss these and the other recent works that may help extend its usefulness in an accompanying News and Views (15146).

CRISPR-Cas is but one example of a technique arising from one area of science whose applications reach well beyond the boundaries of that original discipline. For example the 'CLARITY' imaging technique, in which tissues are fixed in an acrylamide mesh and stripped of their plasma membranes, was originally developed for neuroscience but has now been adapted for plant tissues (W. M. Palmer et al., Sci. Rep. 5, 13492; 2015). Plant-enzymeassisted (PEA)-CLARITY allows deep optical visualization of stains, expressed fluorescent proteins and IgG-antibodies in plant leaves. The adaption of the approach for plant tissue required additional digestion with plant derived enzymes to increase penetration of antibodies into plant tissues.

Despite the synergistic relationship between technical advances and conceptual breakthroughs it can often seem that the latter garner a disproportionate amount of glory. There are of course a number of journals whose raison dêtre is to publish advances in methodology. Our sister journal Nature Methods is one such who earlier this year published on the development of a set of fluorescent reporters for semi-quantitative detection of auxin responses which, although developed in Arabidopsis thaliana, should be easily adapted for other plant species (C.-Y. Liao et al., Nature Methods 12, 207-210; 2015).

Another of our sister journals, Nature Protocols, concentrates on detailed descriptions of proven methodologies whose snags and pitfalls have been sufficiently worked out that they can be adopted by any lab, not just the handful that developed them. Among the protocols published in the last few months has been a metabolomics approach that uses mass spectrometry to analyse the contents of single cells within living plant tissue (T. Fujii et al., Nature Protoc. 10, 1445-1456; 2015). There is also a description of how whole-mount immunolocalization can be used to follow the subcellular localization of proteins during female meiosis and differentiation of the megaspore (R. Escobar-Guzmán et al., Nature Protoc. 10, 1535-1542; 2015).

Despite the excellence of methods and protocols journals, there is an argument that such singular and essentially static descriptions of a methodology cannot successfully capture the essential nature of scientific investigation. Experimentation is more like cookery; every lab and even every researcher has their own favoured recipe for even the most basic of laboratory activities, and their way of preserving the details is often on stained and fading pieces of paper in dog-eared notebooks. Also like cookery, the Web is proving to be a convenient way to capture and share this 'common' knowledge gained through the personal experience of individual practitioners.

Many labs post details of the protocols that they use on their university or departmental web pages, while equipment and reagent suppliers frequently supply protocols for the use of their products through their websites. However, it can be difficult to find the description of a particular technique when searching such varied locations so sites that try to simplify matters by aggregating this content and encouraging more centralized deposition have arisen.

The Protocol Exchange and SpringerProtocols deserve a mention in this context but there are a number of others. Indeed one of the earliest ventures into creating an online community for scientists, Open WetWare was founded in 2005 at MIT to "promote the sharing of information, know-how, and wisdom among researchers and groups who are working in biology and biological engineering"; lab protocols are an important part of achieving it.

Open WetWare has considerable strength in synthetic biology but it is not heavily used by plant biologists. Perhaps a resource more tightly focussed on the plant community is needed. That is certainly the belief behind Plantae a joint venture between the American Society of Plant Biologists and the Global Plant Council, which aims to be "a robust digital ecosystem where connections, content, tools and services help researchers, students, industry professionals and educators thrive". The exchange of methodologies and experimental knowhow will doubtless be an important part of what Plantae becomes, but it is unlikely that it will ever fully replace the conference bar as a venue for swapping tips and tricks with colleagues. 